\title{
Triboelectric Nanogenerators as a New Energy Technology: from Fundamentals, Devices, to Applications
}

\author{
Guang Zhu ${ }^{1, *}$, Peng, Bai ${ }^{2}$, Jun Chen ${ }^{2}$, Qingshen Jing ${ }^{2}$, Zhong Lin Wang ${ }^{1,2, *}$ \\ ${ }^{1}$ Beijing Institute of Nanoenergy and Nanosystems, Chinese Academy of Sciences, Beijing, \\ 100083, China. \\ ${ }^{2}$ School of Materials Science and Engineering, Georgia Institute of Technology, Atlanta, GA \\ 30332, USA.
}

*Correspondence to: zhuguang@binn.cas.cn, zlwang@binn.cas.cn

Keywords: contact electrification, self-powered electronics, energy harvesting, triboelectric nanogenerator

\begin{abstract}
Contact electrification is coupled with electrostatic induction in developing triboelectric nanogenerator as a new energy technology. The triboelectric nanogenerator has two basic operating modes that can be used to harvest a variety of mechanical energy. It provides not only a viable means of powering portable and wearable electronics, but also demonstrates a possible route towards power generation in large scale. This paper makes a comprehensive review on fundamentals, operating modes, device design and performance enhancement of this newly emerged technology.
\end{abstract}

\section{Introduction}

The advance of integrated circuits in the last decade has ushered in the age of miniaturized electronics that are becoming more portable, wearable, stand-alone and even implantable than ever before. They then require power solutions that are sustainable, maintenance-free and even 
perpetual, making the use of conventional power supplies such as battery limited. With this regard, energy harvesting techniques that can capture and convert ambient energy have emerged as a supplementary and even an alternative power solution ${ }^{1-4}$. Mechanical energy, due to its abundant availability, is an ideal source for energy harvesting. Well-established transduction mechanisms for mechanical energy harvesting include electrostatic, electromagnetic, and piezoelectric effects, which have been extensively studied for a few decades ${ }^{5-13}$.

In the last several years, a new type of energy harvesting technology named as triboelectric nanogenerator (TENG) has emerged to harness ambient mechanical motions ${ }^{14-17}$. The TENG has a novel and unique mechanism by using the coupling between triboelectric effect and electrostatic induction. It features unparalleled advantages over other developed existing technologies, including high power density, light weight, small size, low cost, flexibility and even transparency ${ }^{18,19}$. Since its first report in 2012, it has evolved very rapidly and attracted extensive research interests on a global scale. Basic principles and modes have been proposed; various device structures for harvesting a broad range of mechanical energy have been reported; output power has been dramatically promoted through advanced device designing; and attractive applications of this technology in energy and sensing have been demonstrated.

In this review, we will first have a concise discussion on fundamentals of the physical model of the TENG, which will be followed by an introduction of the basic operating modes. Then we will have detailed elaboration on how to develop high-performance devices. Our recent works will be shown to illustrate each designing strategy. Challenges and prospects will be presented in the end.

\section{Fundamentals}


Triboelectric effect is a type of charge transfer by which any two materials, after contact with each other, become electrically charged in opposite signs. The origin of this effect is still shrouded in unsettled questions; and the type of the charge species is still under debate ${ }^{20-24}$. However, it is known that the triboelectric charges are only confined on the surface of materials. They neither recombine nor get annihilated. Instead, they stay in a quasi-permanent way for an extended period of time although minor charge migration occurs ${ }^{25,26}$. The effect has been utilized in applications including electrostatic separations, self-assembly, photocopy and laser printing ${ }^{27-}$ ${ }^{29}$. It is because triboelectric charges are immobile and difficult to be conducted away that triboelectric effect has not been applied in power generation, though it was used to produce high voltage.

The key innovation of the TENG is to incorporate electrostatic induction into an electricitygenerating process. Conductive electrodes that can provide mobile charges are fabricated on the back side of the triboelectric charges. In a TENG, it is induced charges on the back electrodes instead of triboelectric charges that actually form the output current. When two pieces of materials carrying opposite triboelectric charges make relative motion, electric potential difference created between the two electrodes causes transient flow of induced charges. As a result, electric current is produced, delivering effective output power to a load if connected between the electrodes. In this process, mechanical energy is thus converted into electrical energy. The form of the relative motion can be diverse, making the TENG capable of harvesting various types of mechanical energy.

\section{Device structures}

\subsection{Contact mode}




\subsubsection{Basic energy-generation process}

The contact mode features a motion direction that is perpendicular to the charged surfaces in a TENG. Our work in 2012 was the first to propose an accurate and clear description of the energy-generation process ${ }^{15}$. Polymethylmethacrylate (PMMA) and polyimide (Kapton) were used as a pair of contact materials.

In an open-circuit condition, exchange of induced charges cannot occur between the two electrodes. Shown in Fig. 1, no triboelectric charges are generated in the original state (Fig. 1a I). Due to an externally applied force, the two materials are brought into contact. Surface charge transfer then takes place because of triboelectric effect ${ }^{30-32}$. According to the triboelectric series, negative charges are injected from PMMA into Kapton, leaving positive charges on the PMMA surface (Fig. 2a II). As the PMMA layer moves away, an electric potential difference (equivalent to open-circuit voltage) is then established between the two electrodes. It can be expressed by the following equation.

$V_{o c}=\frac{\sigma d}{\varepsilon_{0}}$

where $\sigma$ is the triboelectric charge density, $\varepsilon_{o}$ is the vacuum permittivity, and $d$ is the gap between the two contact materials. When the PMMA layer moves backward, the open-circuit voltage diminishes and drops back to zero when a complete contact is made again (Fig. 1a V and 1a VI). It can be derived that the open-circuit voltage is dependent on the relative position of the two charged layers. If they are held still, the open-circuit voltage will keep stable.

In a short-circuit condition, electrons can redistribute between the two back electrodes due to electrostatic induction, making net induced charges possible on a single electrode. The induced charge density $\left(\sigma^{\prime}\right)$ can be expressed as below. 
$\sigma^{\prime}=\frac{\sigma \mathrm{d} \varepsilon_{\mathrm{rk}} \varepsilon_{\mathrm{rp}}}{\mathrm{d}_{1} \varepsilon_{\mathrm{rp}}+\mathrm{d} \varepsilon_{\mathrm{rk}} \varepsilon_{\mathrm{rp}}+\mathrm{d}_{2} \varepsilon_{\mathrm{rk}}}$

where $\varepsilon_{r k}$, and $\varepsilon_{r p}$ are the relative permittivity of Kapton and PMMA, respectively, $d_{1}$ and $d_{2}$ are the thickness of the Kapton film and the PMMA layer, respectively. The flow of the induced charges then forms output current whose direction relies on the mechanical motion direction. Therefore, reciprocating motion can produce alternating current.

\subsubsection{Energy harvesting from direct force}

It is apparent that the contact mode applies to any direct force, including pressing, impact, shock, etc. In one of our demonstrations, foot stomp was harnessed as the source of mechanical excitation $^{16}$. The TENG has a layered structure with two substrates, as schemed in Fig. 2a. Polymethyl methacrylate (PMMA) was selected as the material for substrates. On the bottom part, a layer of contact electrode is prepared. The contact electrode plays dual roles of electrode and contact surface. It consists of a gold thin film and gold nanoparticles coated on the surface. Fig. $2 b$ shows uniform distribution of the nanoparticles on the surface. On the top part, a thin film of gold is laminated between the substrate and a layer of polydimethylsiloxan (PDMS). This electrode is called back electrode. The two substrates are connected by four springs installed at the corners, leaving a narrow spacing between the contact electrode and the PDMS. These springs provide a restoring force that enables repeated contact and separation of the two parts under an external impact. The as-fabricated TENG is exhibited in Fig. 2c.

Triggered by the human stomp, the TENG generated current pulses that had amplitude as high as $2 \mathrm{~mA}$. It could instantaneously light up as many as 600 LED lamps (Fig. 2d and 2e). The foot stomp and light emission were synchronized, indicating that the TENG was a real-time power 
source. The successful demonstration indicates other immediate applications, such as selflighting shoes and self-lighting tiles which can be luminous upon footfalls.

\subsubsection{Energy harvesting from inertial force}

The relative motion between the two charged materials can be also driven by an inertial force, providing a route to making use of vibrational energy. In this regard, a harmonic-resonator-based TENG was developed ${ }^{33}$. It also consists of two separate parts that are connected by springs at corners. If one part is fixed to a vibration source and the other is left freestanding, they will then have repeated contact and separation. As a result, alternating voltage and current are produced, as shown in Fig. 3a and 3b, respectively. The electric output is dependent on the vibration efficiency, which reaches its maximum at the resonant frequency (Figs. 3c and 3d). The resonant frequency can be tuned by parameters such as the mass of the free-standing component and the elastic modulus of the springs.

Several practical demonstrations were made to show the applicability of the harmonicresonator-based TENG. First, shown in Fig. 3e, the TENG was mounted onto an automotive engine. It sucessfully harvested vibrational energy from the operating engine and powered about 20 LED bulbs simultaneously. Second, TENG, sitting on a table, generated electricity and drove small electronics as impact from a nearby human palm initiated vibration of the table (Figure 3f). These deonstrations prove that the TENG is sensitive to small ambient vibrations, making it suitable to a wide range of circumsntacnes for either energy-harvesting or sensing purposes, e.g. highways, bridges, and tunnels.

\subsection{Sliding mode}

3.1.1. Basic energy-generation process 
Two charged surfaces can also make relative motion that is parallel to the charged surface plane17. The basic structure of the sliding TENG is sketched in Fig. 4a. It has a structure in which two contacting surfaces that can slide smoothly with one against the other. On one of the substrates, aluminum thin film plays dual roles of an electrode and a sliding surface. On the other substrate, copper electrode is sandwiched between the substrate and a PTFE film. The PTFE film acts as another sliding surface. At original position, the two sliding surfaces are fully aligned. Driven by a tangential force applied to a substrate, relative displacement in contact mode occurs in lateral direction. After the two surfaces are fully displaced, the reciprocating force retraces them back to the aligned position (Fig. 4b).

Once the PTFE film is brought into contact with the aluminum thin film, surface charge transfer takes place due to the triboelectric effect. At aligned position, positive triboelectric charges on aluminum are fully compensated by the negative counterparts on PTFE, producing no electric field in surrounding space if the electric field at the edge is ignored (Fig. 4c). Once a relative displacement is introduced, triboelectric charges that are not compensated at the displaced/mismatched areas result in an effective dipole polarization parallel to the direction of the displacement. Therefore, an electric potential difference is produced across the two electrodes, as illustrated by a simulation plot via COMSOL in Fig. 4d. Thus, the open-circuit voltage can be analytically expressed as a function of the displacement.

$V_{o c}=\frac{q}{2 \pi \varepsilon_{0} W L}\left[\frac{l}{2} \ln \left(t^{2}+l^{2}\right)+t \tan ^{-1}\left(\frac{l}{t}\right)\right]$

where $l$ is the mismatched displacement between the two sliding plates, $q$ is the quantity of the triboelectric charges on one plate, $\varepsilon_{0}$ is the vacuum permittivity, $L$ is the length of the TENG's plate, $W$ is the width of the TENG, and $t$ is the thickness of the PTFE film. The theoretically calculated $V_{o c}$ fits the trend of the experimental data (Fig. 4e). These two sets of results both 
reveal that the $V_{o c}$ drastically increases once the displacement occurs and quickly reaches a nearly saturated value.

If the two electrodes are electrically connected, once displacement is established, the uncompensated negative triboelectric charges on PTFE will repulsively drive free electrons on the copper electrode to the aluminum electrode $\left(\right.$ Fig. $\left.4 \mathrm{f}_{2}\right)$. The flow of induced electrons lasts until the displacement reaches the maximum. At the fully displaced position, the positive triboelectric charges are completely balanced out $\left(\right.$ Fig. $\left.4 \mathrm{f}_{3}\right)$. As the displacement is diminished by the reciprocating force, the induced electrons flow back to the copper electrode (Fig. $4 \mathrm{f}_{4}$ ) until the fully aligned position is restored (Fig. $4 \mathrm{f}_{1}$ ). Therefore, in the entire process, alternating current is produced through the external load.

\subsubsection{Harvesting energy from linear motion}

Based on the basic operating principle of the sliding mode, a case-encapsulated TENG was developed $^{34}$. The structural configuration is depicted in Fig. 5. It has two concentric cylinders that can slide against each other. The case wrap applied tightly around the outer cylinder allows the two cylindrical structures to have relative motion in a coaxial one-dimension. Polyimide was selected as an electrification material for generating the triboelectric charges and also as the supporting substrate. On both sides of the polyimide film, copper stripes were deposited with a periodic pitch. The electrode patterns on different sides exhibited a linear shift of half pitch so that they are complementary to each other. Two polyimide films having the same electrode patterns formed a pair of contact surfaces. They are adhered onto the outer surface of a PMMA tube and the inner surface of a foam tube, respectively (Fig. 5d). The fully assembled CE-TENG is shown in Fig. 5e. 
The device was used to harvest random body motions. By simply shaking the CE-TENG, multiple LED white bulbs connected in parallel were lighted up (Figs. 5e and 5f). The CE-TENG can be even attached to certain parts of the human body, such as hand, forearm, leg and even torso. Its casing-encapsulated structure allows linear motions between the two parts during normal human movements such as running and jumping.

\subsubsection{Harvesting energy from rotation}

A coaxial cylindrical structured rotating-TENG (Fig. 6) was developed to harvest mechanical energy from rotation ${ }^{35}$. A TENG with a total contact area of $12 \mathrm{~cm}^{2}$ contains multiple strip units that are connected in parallel. For a TENG with 8 strip units at a linear rotational velocity of 1.33 $\mathrm{m} / \mathrm{s}$, the instantaneous short-circuit current and the open-circuit voltage could reach $90 \mu \mathrm{A}$ and $410 \mathrm{~V}$, respectively, corresponding to an instantaneous maximum power density of $36.9 \mathrm{~W} / \mathrm{m}^{2}$ and an equivalent average direct current of $45.6 \mu \mathrm{A}$. Higher output power can be achieved by using a higher density of strip units and/or applying larger linear rotational velocity. Since rotation is one of the most common forms of motion in ambient environment, a fully packaged cylindrical rotating TENG has potential applications in harsh environment, outdoors or even under water to harvest energy from water flow.

\section{High-performance device}

\subsection{Materials issues}

The output voltage as well as the output current is linearly related to the density of triboelectric charges that is largely defined by materials. The triboelectric series is a list of materials that are arranged according to their tendency in gaining or losing electrons. Materials 
that are listed on the top tend to become positively charged while those listed at the bottom are more likely to gain negative charges. Therefore, the materials used for the two contact surfaces in TENG need to have the largest possible separation on the triboelectric series. Besides, these materials need to be compatible to thin-film structure, excluding some of the materials even though they may excel in producing triboelectric charges. Generally, positively charged materials used in a TENG usually include Polyamide and metal, while those negatively charged materials are mostly fluorinated polymers, such as PTFE, FEP and PVDF.

In order to further increase the surface charge density, we demonstrated an approach that took advantage of the dipole moment in polarized polyvinylidene fluoride (PVDF), substantially enhancing the output power density of the TENG. In this work, polarized PVDF was used as the electrification material $^{36}$. The electric output was dependent on the polarization direction of the PVDF. Forward-polarized PVDF could greatly enhance the output power, while reversepolarized PVDF resulted in reduced output power, as shown in Fig. 7. The enhancement reached as high as $240 \%$ under a constant force of $50 \mathrm{~N}$. The reason for this modulation was proposed to be the intrinsic dipole moment of the PVDF that altered the surface potential level of the PVDF film. As a result, the surface charge transfer between the PVDF and another contact material was modulated.

\subsection{Device structures}

\subsubsection{Macro-structure}

We developed an innovative design of a TENG with multiple layers of units fabricated on a single flexible substrate (Fig. 8$)^{37}$. The structure provides a novel and simple method to stack multiple TENGs without either expanding the area or considerably complicating the fabrication

process. As a consequence, we were able to achieve multi-folds enhancement of the output 
power while keeping the TENG's area constant. A TENG with a size of $3.8 \mathrm{~cm} \times 3.8 \mathrm{~cm} \times 0.95$ $\mathrm{cm}$ contains 5 active layers of units that are parallel connected. The instantaneous short-circuit current and the open-circuit voltage could reach $0.66 \mathrm{~mA}$ and $215 \mathrm{~V}$, corresponding to an instantaneous power density of $9.76 \mathrm{~mW} / \mathrm{cm}^{2}$ and $10.24 \mathrm{~mW} / \mathrm{cm}^{3}$ at device level.

\subsubsection{Micro-structure}

When we first proposed the basic sliding mode, a concept of linear gratings was demonstrated to enhance the current amplitude, current frequency, and conversion efficiency ${ }^{17}$. Identical linear grating with uniform period is fabricated on both sliding surfaces. Every row of the grating units can be considered as a reduced-sized TENG; and it is in parallel connection with all other rows. In contrast to a non-grating TENG that needs to be fully displaced in order to complete pumping of the induced charges for one time, the grating TENG only requires a displacement of a unit length to completely transport the induced charges. Therefore, for a one-way sliding process across the entire length of the TENG, the induced charges can be pumped for $(2 N-1)$ times in total, where $N$ is the number of grating units. The following equation represents the total induced charges $Q$ in a single sliding across the entire length of the TENG.

$$
\begin{aligned}
Q & =N q^{\prime}+\left|-q^{\prime} \times(N-1)\right|+q^{\prime} \times(N-1)+\cdots+\left|-q^{\prime}\right|+q^{\prime} \\
& =N q^{\prime}+2 q^{\prime} \times \sum_{i=1}^{N-1} i \\
& =\left(2 q^{\prime} N\right) N / 2
\end{aligned}
$$

where $q^{\prime}$ is the induced charges generated from a single grating unit for a displacement of the unit length. As shown in Figs. 9a-9c, the amplitude of short-circuit current produced by TENGs having 6, 8 and 10 grating units increases in a linear manner, as indicated in Eq. (4). Therefore, high-density and fine-sized periodic pattern on the sliding surface is the key to substantially promote the output power of the TENG. 
Following the above grating concept, we developed two types of high-performance TENGs that have fine-sized periodic patterns of different shapes. One of them is based linear microgratings ${ }^{19}$. The device is composed of PTFE thin films with a pair of metal gratings on opposite sides. As schemed in Fig. 10a, the grating is a collection of metal strips separated by equal-sized intervals. The paired gratings are identical but complementary with relative displacement of half pitch (zoom-in view in Fig. 10a). Fig. 10b exhibits a photograph of the PTFE thin film with double-sided metal gratings. On the top surface, a layer of PTFE nanoparticles is applied as surface modification (Fig. 10c). Two of the thin films with different length are prepared, which have a total area of $60 \mathrm{~cm}^{2}$, a total volume of $0.2 \mathrm{~cm}^{3}$, and a total weight of $0.6 \mathrm{~g}$. The relative motion direction is perpendicular to the metal strips. The short-circuit current reaches as high as $2 \mathrm{~mA}$ when sliding at a relative velocity of $2 \mathrm{~m} / \mathrm{s}$ and keeps increasing in a linear way as a function of the velocity (Fig. 10d). The average optimum output power under the matched load is $0.7 \mathrm{~W}$ at the sliding velocity of $0.7 \mathrm{~W}$ and can be further enhanced to almost $3 \mathrm{~W}$ at the sliding velocity of $10 \mathrm{~m} / \mathrm{s}$ (Fig. 10f)

Another shape of the periodic pattern is radial sectors ${ }^{18}$. The device consists of mainly two parts, i.e., a rotator and a stator. The rotator is a collection of radially-arrayed sectors separated by equal-degreed intervals in between. The rotator has a total of 60 units. For the stator, it is divided into three components. A layer of FEP as an electrification material, a layer of electrodes, and an underlying substrate are laminated along the vertical direction. The electrode layer is composed of two complementary-patterned electrode networks that are disconnected by fine trenches in between. Operating at a rotation rate of $3000 \mathrm{r} \cdot \mathrm{min}^{-1}$, a rotary TENG having a diameter of $10 \mathrm{~cm}$ can produce an open-circuit voltage of $\sim 850 \mathrm{~V}$ and a short-circuit current of $\sim 3 \mathrm{~mA}$ at a frequency of $3 \mathrm{KHz}$. Under the matched load of $0.8 \mathrm{M} \Omega$, an average output power of 
$1.5 \mathrm{~W}$ (an area power density of $19 \mathrm{~mW} \cdot \mathrm{cm}^{-2}$ ) can be delivered to an external load at an efficiency of $24 \%$, representing a gigantic leap in terms of output power by orders of magnitude. The TENG is demonstrated as an effective measure in harvesting a variety of ambient mechanical motions, such as light wind (Fig. 11a), water flow (Fig. 11b), and body movement (Fig. 11c). More importantly, a complete power-supplying system is built through integrating a power management circuit with the TENG, which can provide a continuous direct current source at a constant voltage for sustainably driving as well as charging various commercial electronics (Fig. 12).

\section{Prospects and Challenges}

Compared to other existing power-generating technologies, the TENG has high output power as well as light weight and small size because it can be fabricated from thin-film materials. As a result, the TENG is superior in power density in terms of both power-to-volume ratio and powerto-weight ratio. This advantage is especially important for developing self-powered portable/wearable electronics, where size and weight management are important. Furthermore, since the TENG relies on surface charging effect, it only requires very small amount of materials, which are conventional materials that are readily available. Because of the scalable and straightforward fabrication process, the TENG is significantly cost-effective, an unparalleled advantage for potential practical applications. Besides, the broad choices of materials enable unique applications, e.g. those in healthcare where biocompatibility is required.

It must be pointed out that there are some challenges to be overcome for the long-term development of the TENG. First, the TENG can easily produce extremely high voltage but low current, which means that the TENG has very high output impedance. However, conventional electronics usually require high current but low voltage, leading to relatively low input 
impedance. As a result, a TENG and the load have significant mismatch of impedance. Voltage transformers were connected to the TENG in order to increase the current at the expense of the voltage. However, they are normally bulky in size the heavy in weigh, which are unsuitable for a miniaturized self-powered system. Second, the thin-film electrification materials are normally polymers that are susceptible to mechanical wear. Residual particles due to the mechanical wear will prevent the two contact surfaces from intimate contact and thus greatly reduce the output power the TENG. Though dry lubricant made of nanoparticles was used to promote the wearability to some degree, more progress needs to be made in this aspect.

\section{Conclusion}

The triboelectric nanogenerator takes advantage of the coupling effect between contact electrification and electrostatic induction. It provides a practical approach in harvesting mechanical energy in a variety of forms, including impact, vibration, sliding, rotation, etc. This newly emerged technology features high output intensity, light weight and small volume. It is promising to become a viable means of powering small electronics and even a possible solution for large-scale power generation.

\section{Acknowledgements}

Research was supported by U.S. Department of Energy, Office of Basic Energy Sciences, the "thousands talents" program for pioneer researcher and his innovation team, China, and Beijing City Committee of science and technology projects (Z131100006013004, Z131100006013005).

\section{References}


1. S. P. Beeby, M. J. Tudor, N. M. White, Meas. Sci. Technol. 2006, 17, R175.

2. Z. L. Wang, Adv. Mater. 2011, 24, 280.

3. P. D. Mitcheson, E. M. Yeatman, G. K. Rao, A. S. Holmes, T. C. Green, P. IEEE 2008, 96, 1457.

4. J. A. Paradiso, T. Starner, IEEE Pervas. Compu. 2005, 4, 18.

5. P. Miao, P. D. Mitcheson, A. S. Holmes, E. M. Yeatman, T. C. Green, B. H. Stark, Microsyst. Technol. 2006, 12, 1079.

6. S. Round, R. K. Wright, J. Rabaey, J. Comput. Commun. 2003, 26, 1131.

7. S. Priya, J. Electroceram, 2007, 19, 165.

8. S. P. Beeby, R. N. Torah, M. J. Tudor, P. Glynne-Jones, T. O'Donnell, C. R. Saha, S. Roy. J. Micromech. Microeng. 2007, 17, 1257.

9. H.-W. Lo, Y.-C. Tai, J. Micromech. Microeng. 2008, 18, 104006.

10. F. Lu, H. P. Lee, S. P. Lim, Smart Mater. Struct. 2004, 13, 57.

11. L. C. Rome, L. Flynn, E. M. Goldman, T. D. Yoo, Science 2005, 309, 1725.

12. J. M. Donelan, Q. Li, V. Naing, J. A. Hoffer, D. J. Weber, A. D. Kuo, Science 2008, 319, 807.

13. A. Harb, Renew. Energy. 2011, 36, 2641.

14. F.-R. Fan, Z.-Q. Tian, Z. L. Wang, Nano Energy 2013, 1, 328.

15. G. Zhu, C. Pan, W. Guo, C.-Y. Chen, Y. Zhou, R. Yu, Z. L. Wang, Nano Lett. 2012, 12, 4960.

16. G. Zhu, Z.-H. Lin, Q, Jing, P. Bai, C. Pan, Y. Yang, Y. Zhou, Nano Lett. 2013, 13, 847.

17. G. Zhu, J. Chen, Y. Liu, P. Bai, Y. S. Zhou, Q. Jing, C. Pan, Z. L. Wang, Nano Lett. 2013, 13, 2282.

18. G. Zhu, J. Chen, Q. Jing, T. Zhang, Z. L. Wang, Nat. Commun. 2014, 5, 3426. 
19. G. Zhu, Y. S. Zhou, P. Bai, X. Meng, Q, Jing, J. Chen, Z. L. Wang, Adv. Mater. 2014, 26, 3788.

20. A. F. Diaz, J. Guay, IBM J. Res. Dev.1993, 37, 249.

21. D. K. Davies, J. Phys. D: Appl. Phys.1969, 2, 1533.

22. C. B. Duke, J. Appl. Phys. 1978, 49, 315.

23. J. A. Wiles, B. A. Grzybowski, A. Winkleman, G. M. Whitesides, Anal. Chem. 2003, 75, 4859.

24. L. S. McCarty, A. Winkleman, G. M. Whitesides, J. Am. Chem. Soc. 2007, 129, 4075.

25. Y. S. Zhou, Y. Liu, G. Zhu, Z.-L. Lin, C. Pan, Q. Jing, Z. L. Wang, Nano Lett. 2013, 13, 2771.

26. Y. S. Zhou, S. Wang, Y. Yang, G. Zhu, S. Niu, Z.-H. Lin, Y. Liu, Z. L. Wang, Nano Lett. 2014, 14, 1567.

27. B. A. Kwetkus, Part. Sci. Technol. 1998, 16, 55.

28. D. M. Pai, B. E. Springett, Rev. Mod. Phys.1993, 65, 163.

29. B. A. Grzybowski, A. Winkleman, J. A. Wiles, Y. Brumer, G. M. Whitesides, Nat. Mater. 2003, 2, 241-245.

30. G. S. P. Castle, J. Electrostat. 1997, 40, 13.

31. E. Nemeth, V. Albrecht, G. Schubert, F. Simon, J. Electrostat. 2003, 58, 3.

32. M. Lungu, Miner. Eng. 2004, 17, 69.

33. J. Chen, G. Zhu, W. Yang, Q. Jing, P. Bai, Y. Yang, T.-C. Hou, Z. L. Wang, Adv. Mater. 2014, 25, 6094.

34. Q. Jing, G. Zhu, P. Bai, Y. Xie, J. Chen, R. Han, Z. L. Wang, ACS Nano 2014, 8, 3836. 
35. P. Bai, G. Zhu, Y. Liu, J. Chen, Q. Jing, W. Yang, J. Ma, G. Zhang, Z. L. Wang, ACS Nano 2013, 7, 6361.

36. P. Bai, G. Zhu, Z. L. Wang, Nano Research. 2014, 7, 990.

37. P. Bai, G. Zhu, Z.-H. Lin, Q. Jing, J. Chen, G. Zhang, J. Ma \& Z. L. Wang, ACS Nano 2013, $7,3713$. 

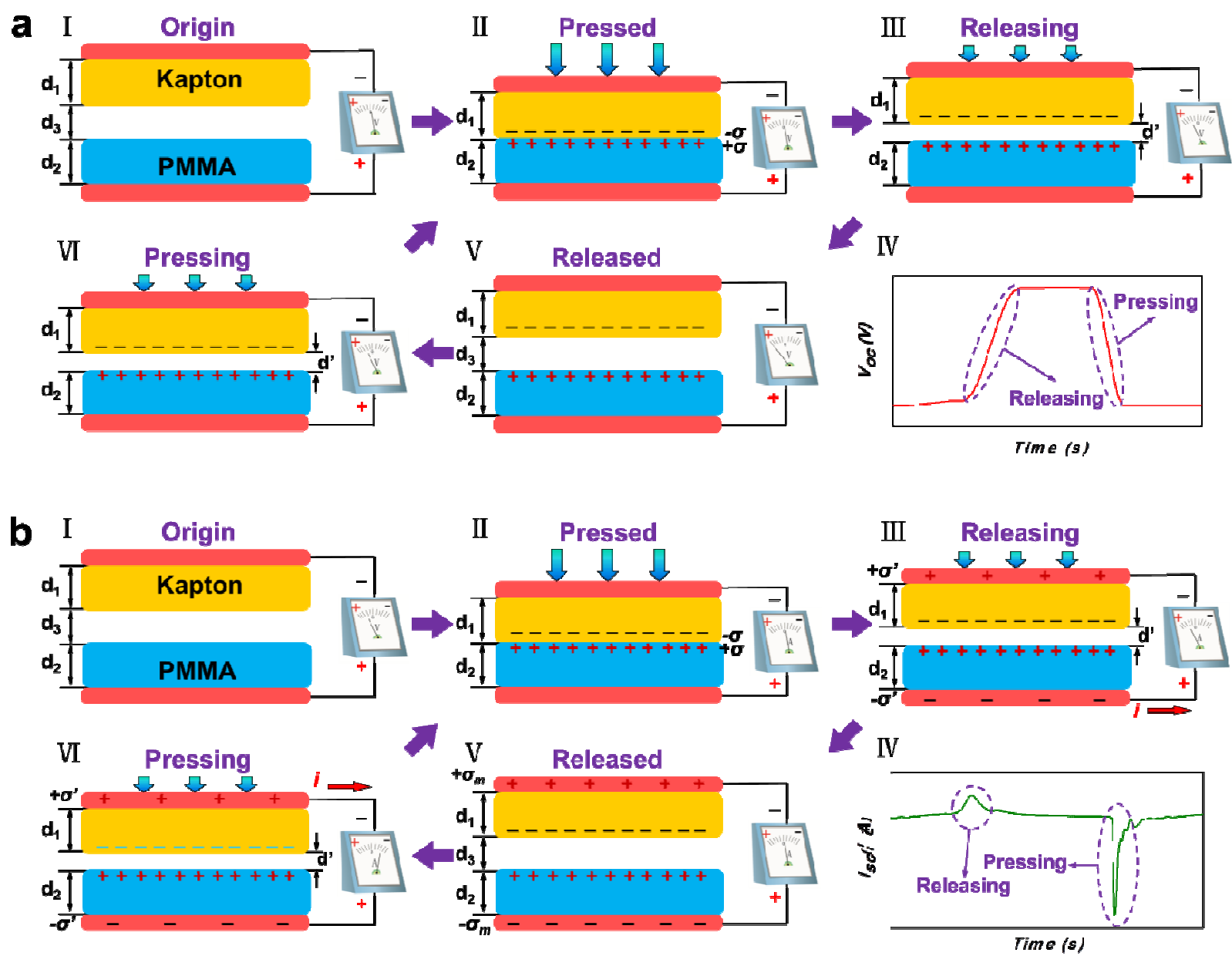

Figure 1. Sketch that illustrates the operating principle of the contact mode. a, Open-circuit condition. b, Short-circuit condition. 

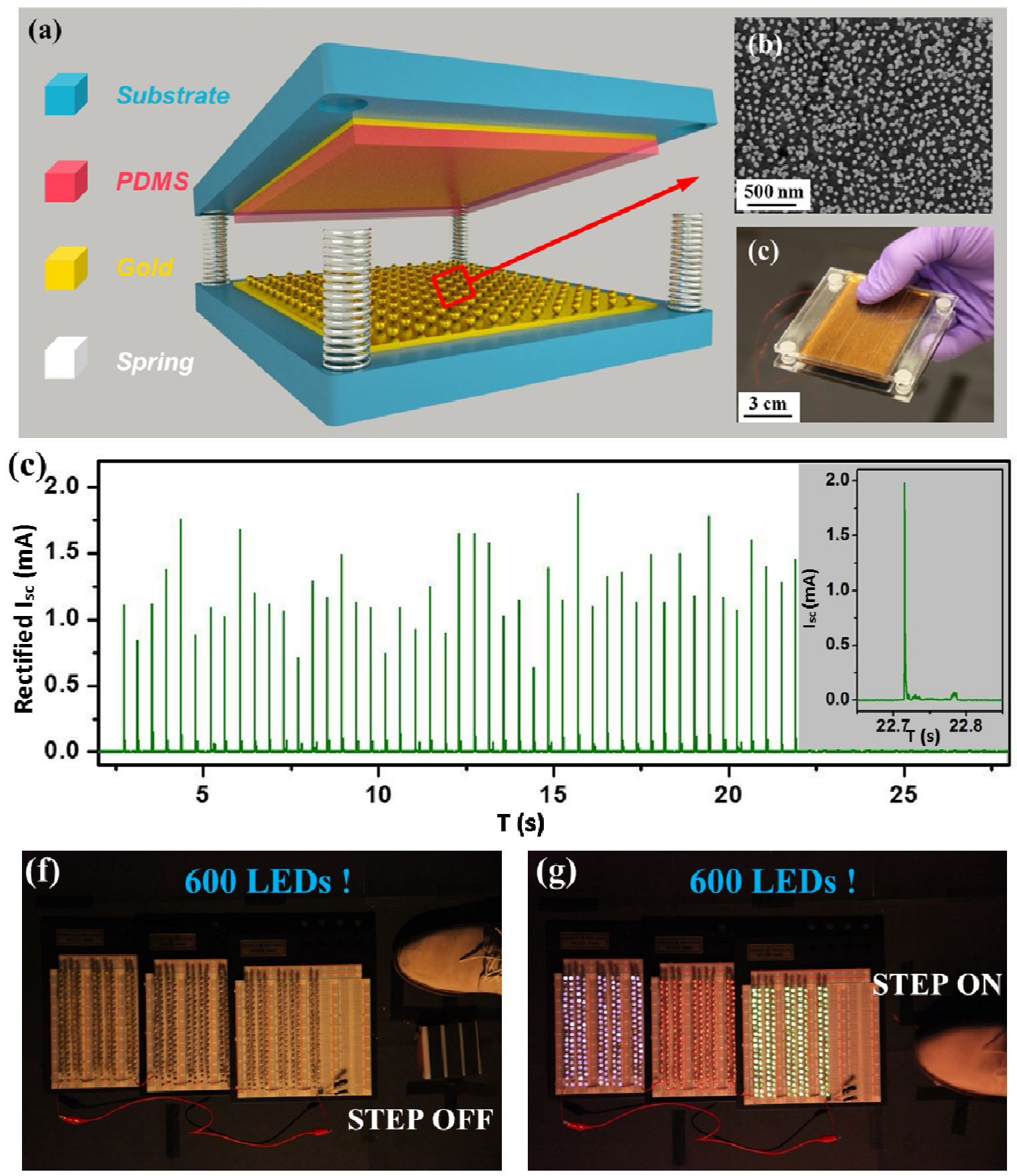

Figure 2. Contact-mode TENG for harvesting energy from impact. (a) Schematic and (b) photograph of a fabricated triboelectric TENG. (c) SEM image of gold nanoparticles coated on gold surface. (d) Short-circuit current when triggered by human footfall that can generate contacting force between $500 \mathrm{~N}$ to $600 \mathrm{~N}$. Inset: enlarged view of the highest current pulse. (e) 
Photograph of a setup in which the TENG acts as a direct power source for 200 commercial green, red and blue LED lamps, respectively and (f) When footstep falls on the TENG, simultaneously lighting up the LEDs in real-time. 

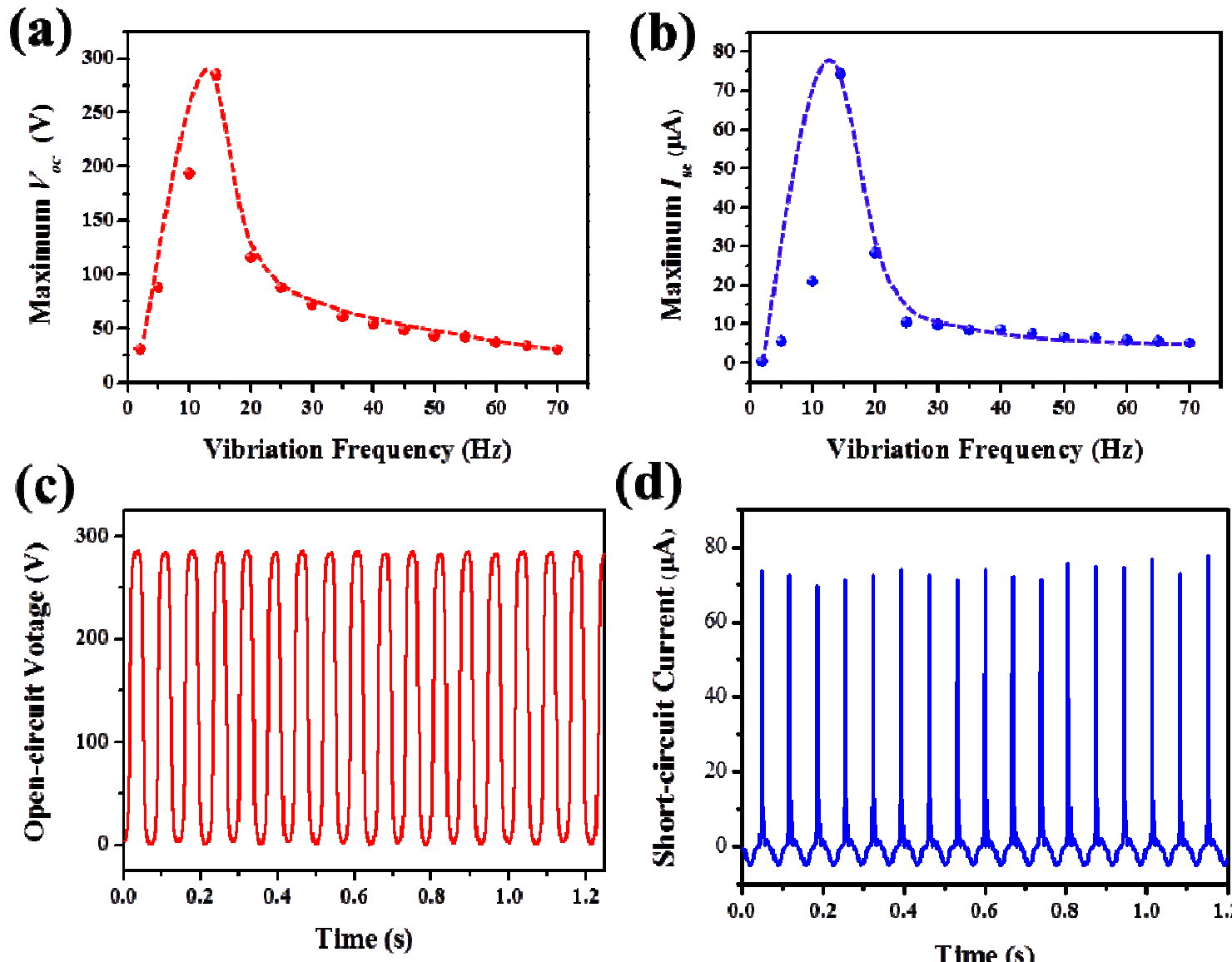

(d)
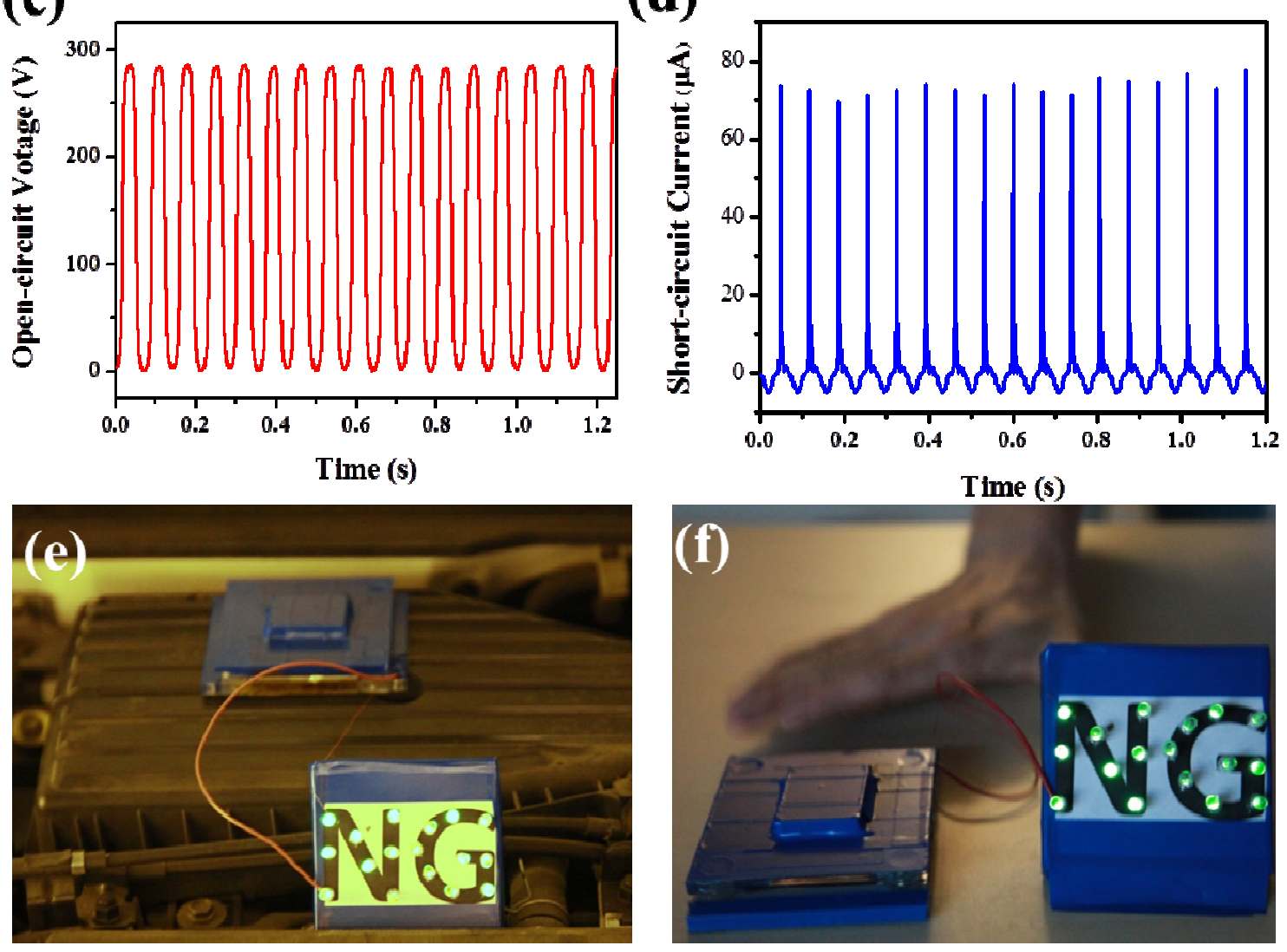

Figure 3. The harmonic resonator TENG based on the contact mode. (a) Open-circuit voltage as a function of vibration frequency. (b) Short-circuit current as a function of vibration frequency. (c) Open-circuit voltage at the resonant frequency of $14.5 \mathrm{~Hz}$. (d) Short-circuit current at the resonant frequency of $14.5 \mathrm{~Hz}$. (e) Photograph that shows a TENG on an automotive engine to 
drive LED bulbs. (f) Photograph that shows a TENG triggered by hand impact on a desk to power LED bulbs. 



Figure 4. Triboelectric generators based on the sliding mode. (a) Schematic of the TENG. (b) A complete sliding cycle of the TENG at different positions. (c) COMSOL simulation result of electric potential distribution when the two surfaces are fully aligned. (d) COMSOL simulation result of electric potential distribution when the two surfaces are halfway displaced. (e) Electric potential difference between the aluminum electrode and the copper electrode as a function of the displacement. Red dots and blue dots represent experimental values and analytical values, 
respectively. (f) A cycle of electricity generation process for illustrating the electricity generation process. 


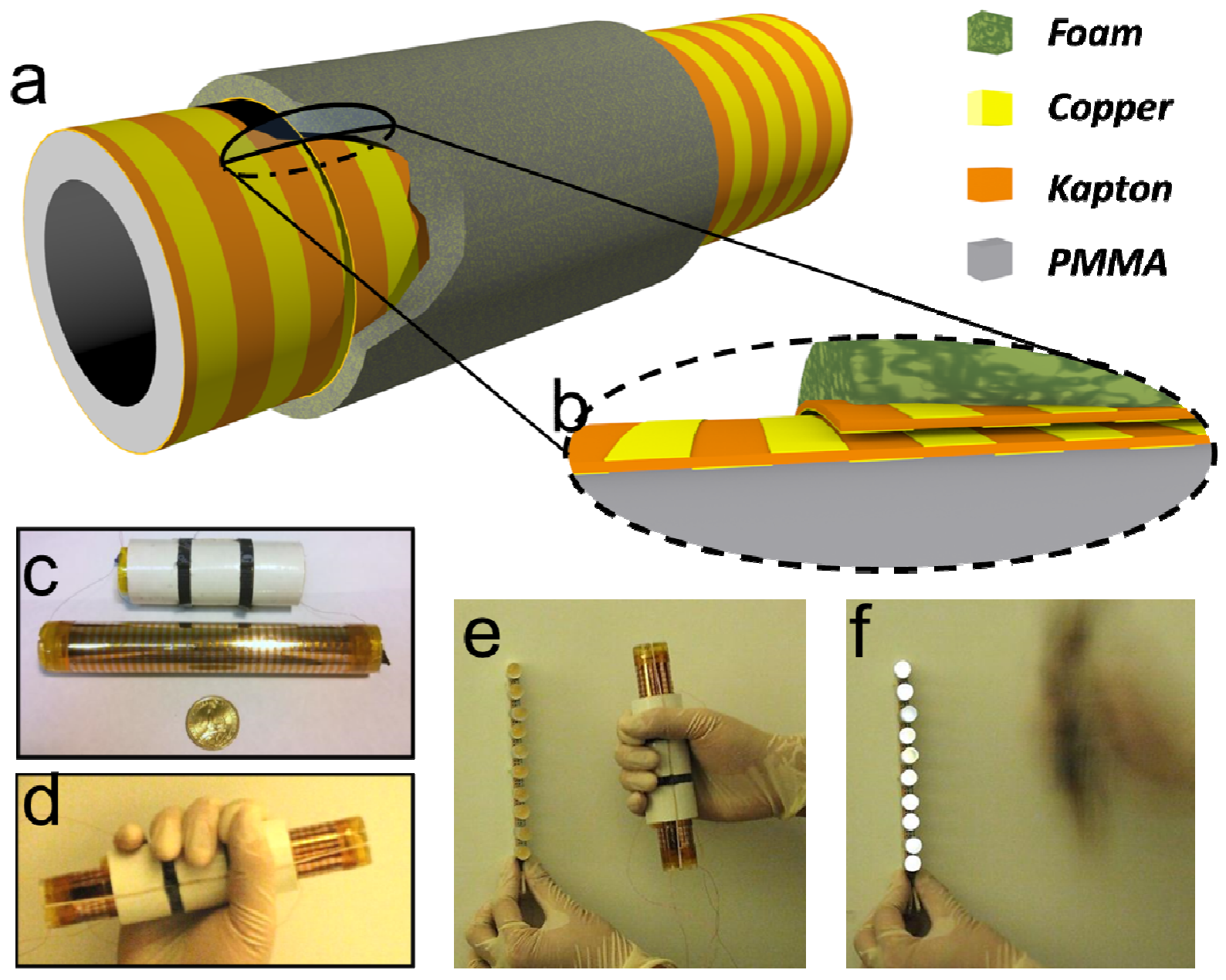

Figure 5. Case-encapsulated triboelectric nanogenerator. (a) 3D model and (b) cross sectional view. A TENG (c) before and (d) after assembly. (e) and (f) The TENG that harvesters human body motion powers white LED bulbs. 

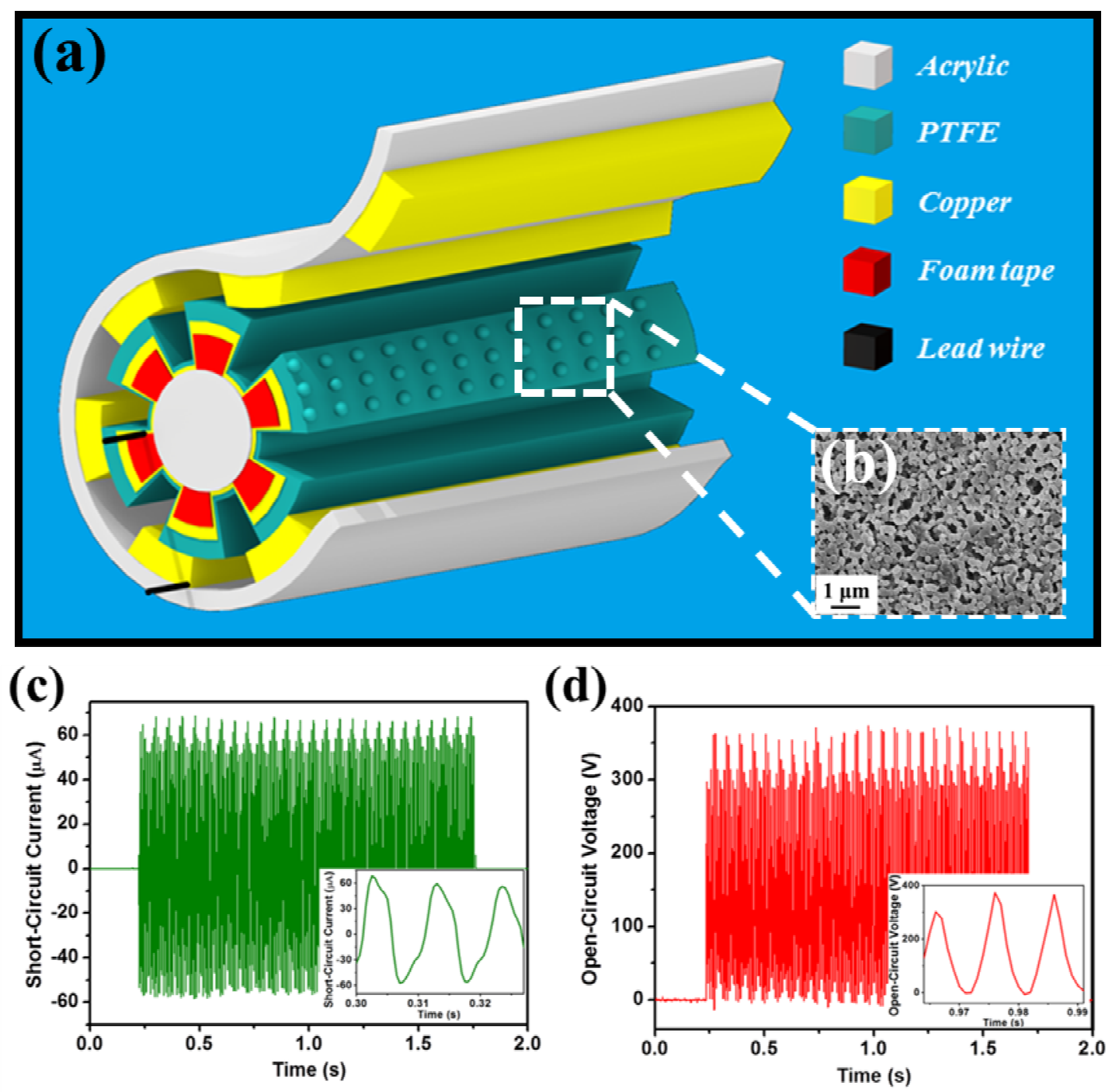

Figure 6. (a) Schematic of the rotating-TENG with 6 strip units. (b) SEM image of PTFE nanoparticles on the surface of PTFE film. (b) Short-circuit current of a rotating-TENG at a linear rotational velocity of $1.33 \mathrm{~m} / \mathrm{s}$ (a rotation rate of $1000 \mathrm{r} / \mathrm{min}$ ). Inset: enlarged view of the current peaks. (b) Open-circuit voltage of a rotating-TENG at a linear rotational velocity of 1.33 $\mathrm{m} / \mathrm{s}$ (a rotation rate of $1000 \mathrm{r} / \mathrm{min}$ ). Inset: enlarged view of the voltage peaks. 
(a)

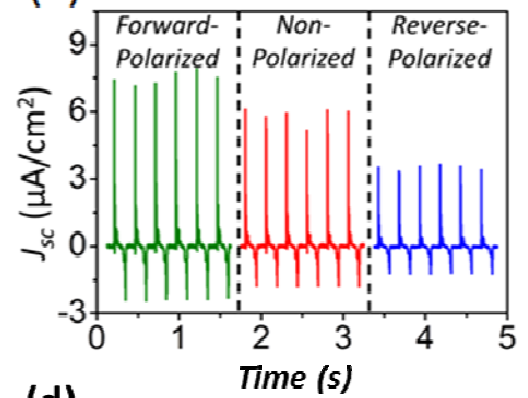

(d)

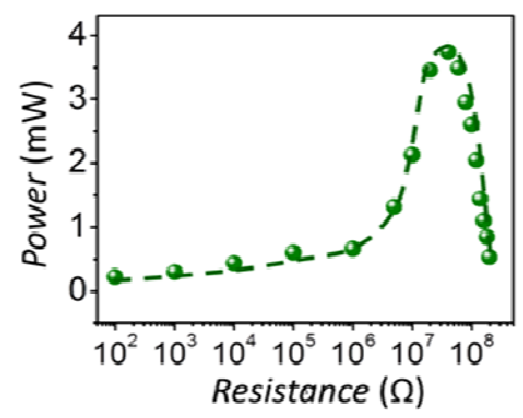

(b)

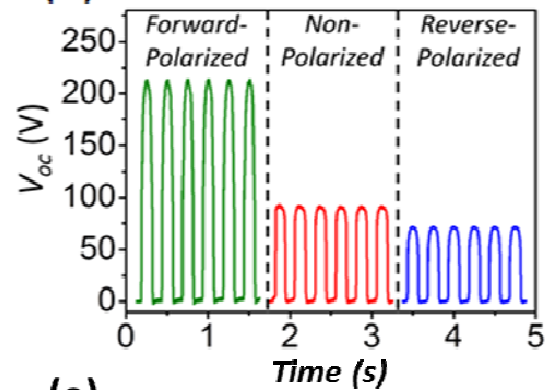

(e)

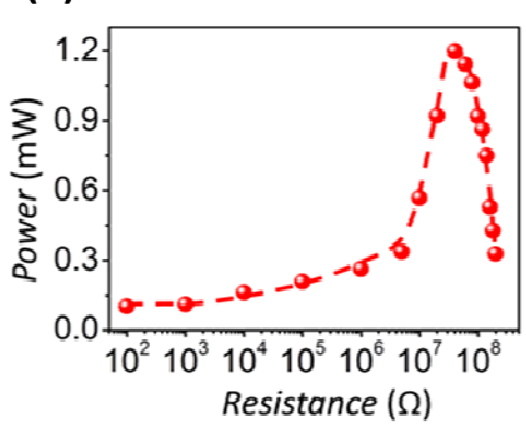

(c)

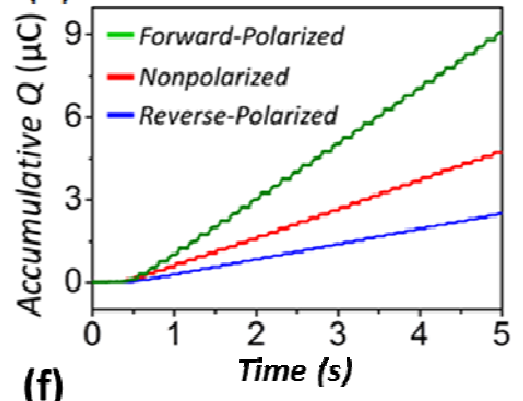

(f)

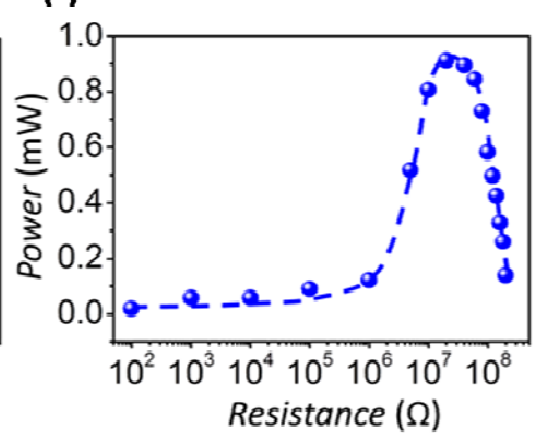

Figure 7. Density of short-circuit current (a), open-circuit voltage (b) and accumulative charge

(c) generated by TENGs fabricated by different types of PVDF films under a periodical compressive force around $50 \mathrm{~N}$. Dependence of the peak power output of the forward-polarized (c), non-polarized (e) and reverse-polarized (f) TENGs on the resistance of the external load. 
(a)

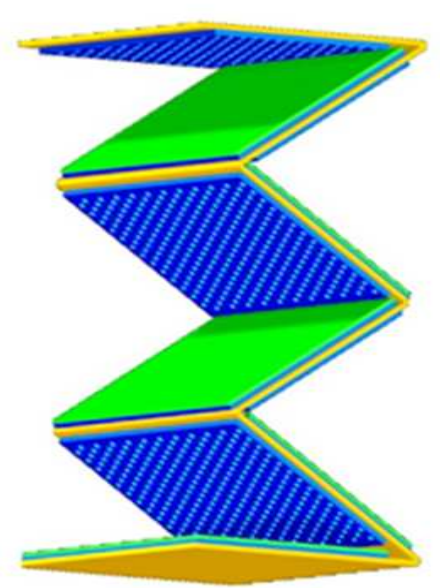

front
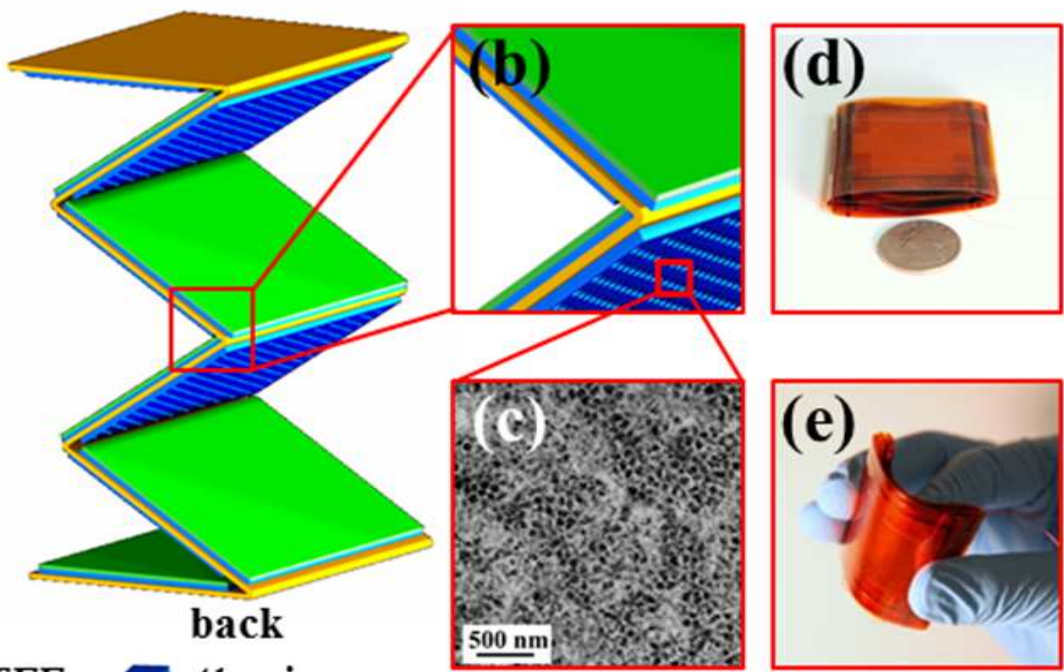

Figure 8. Structure and photographs of a flexible multilayered TENG with 5 layers of units. (a)

Schematic and (b) an enlarged view of the zigzag-shaped structure of the TENG. (c) SEM image of nano-pores on aluminum foils. (d) Photograph of a fabricated flexible multilayered TENG and (e) photograph of a bent flexible multilayered TENG by human fingers. 

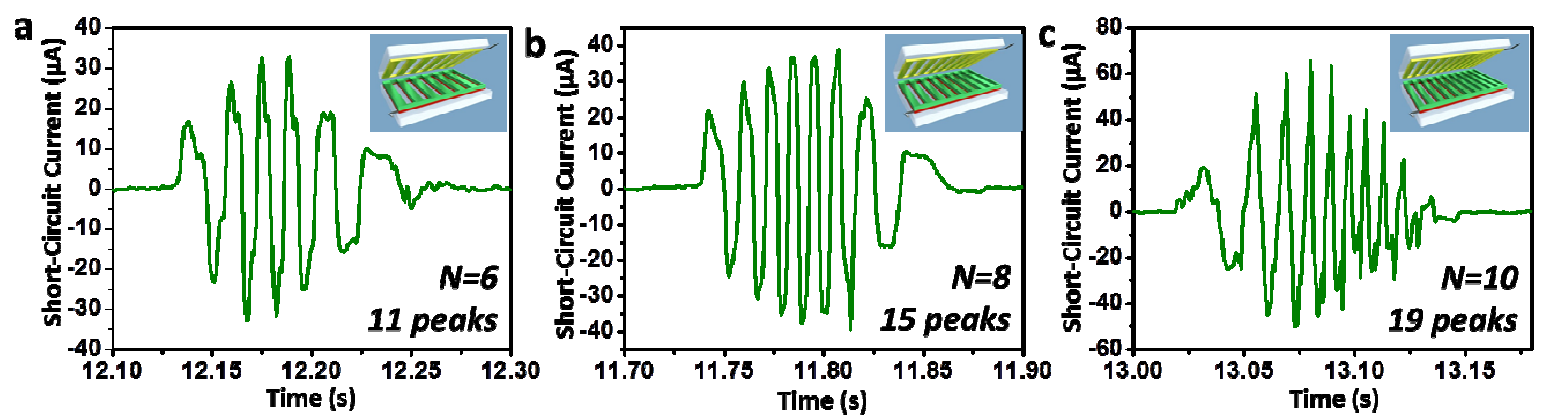

Figure 9. Electrical measurement results of grating TENGs with multiple grating units within a fixed total length. Short-circuit current of a grating TENG with 6 (a), 8 (b) and 10 (c) grating units for a single sliding process across the full width of the TENG. Insets: schematic of the TENGs structure. 
(a)

)
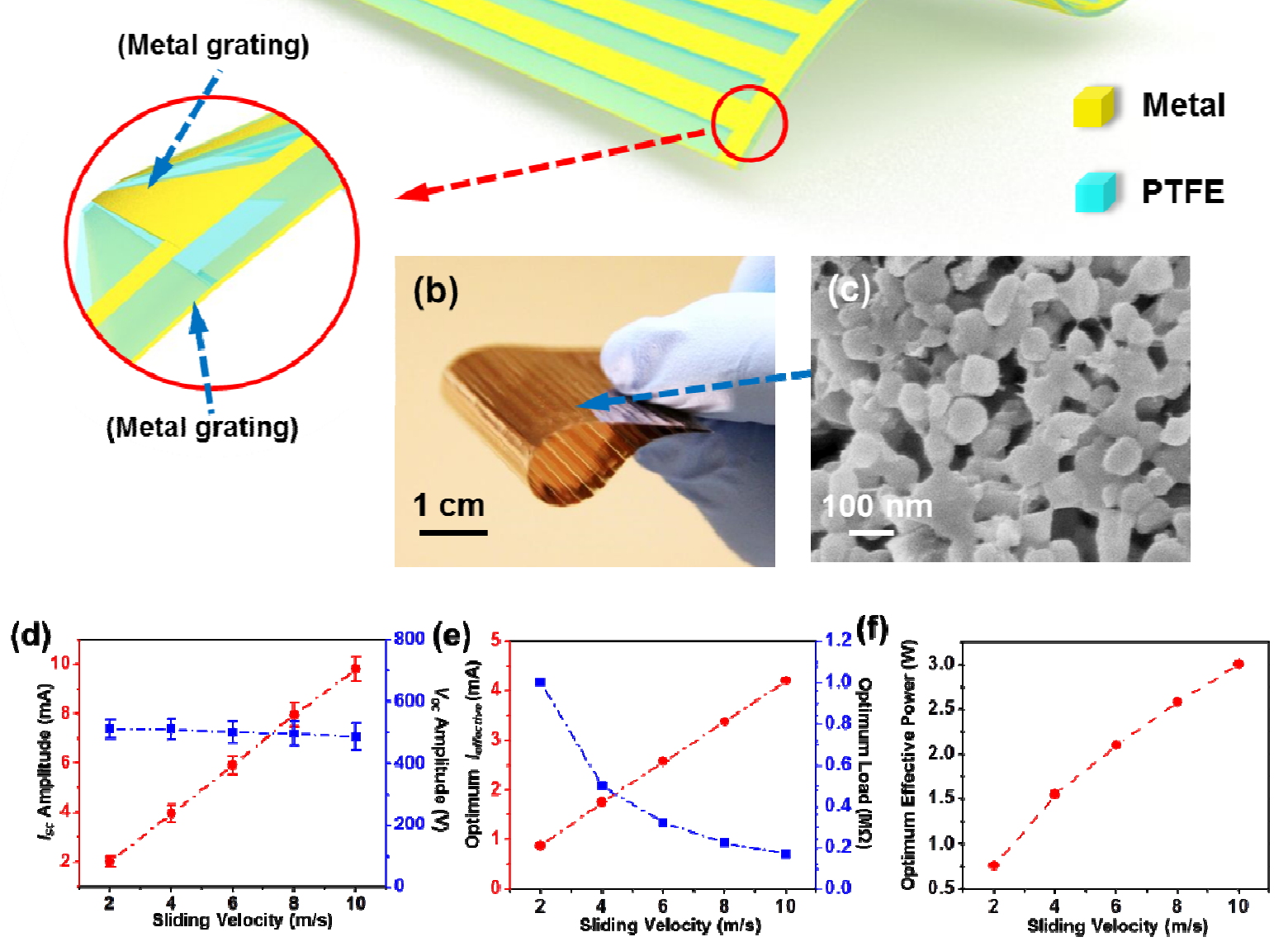

Figure 10. Device design of the linear micro-grating TENG. (a) Schematic illustrations of the thin film that composes of the TENG. The zoom-in illustration (bottom left) reveals that the film consists of a PTFE layer and a pair of metal gratings that have complementary patterns. (b) Photograph of a flexible PTFE film with a pair of complementary metal gratings on both sides. (c) SEM image of PTFE nanoparticles applied onto the surfaces on which friction takes place. (d) Amplitude of short-circuit current and open-circuit voltage with varying sliding velocity. (b) The 
optimum effective current and corresponding load with varying sliding velocity. (c) The optimum effective power of the TENG with varying sliding velocity. 

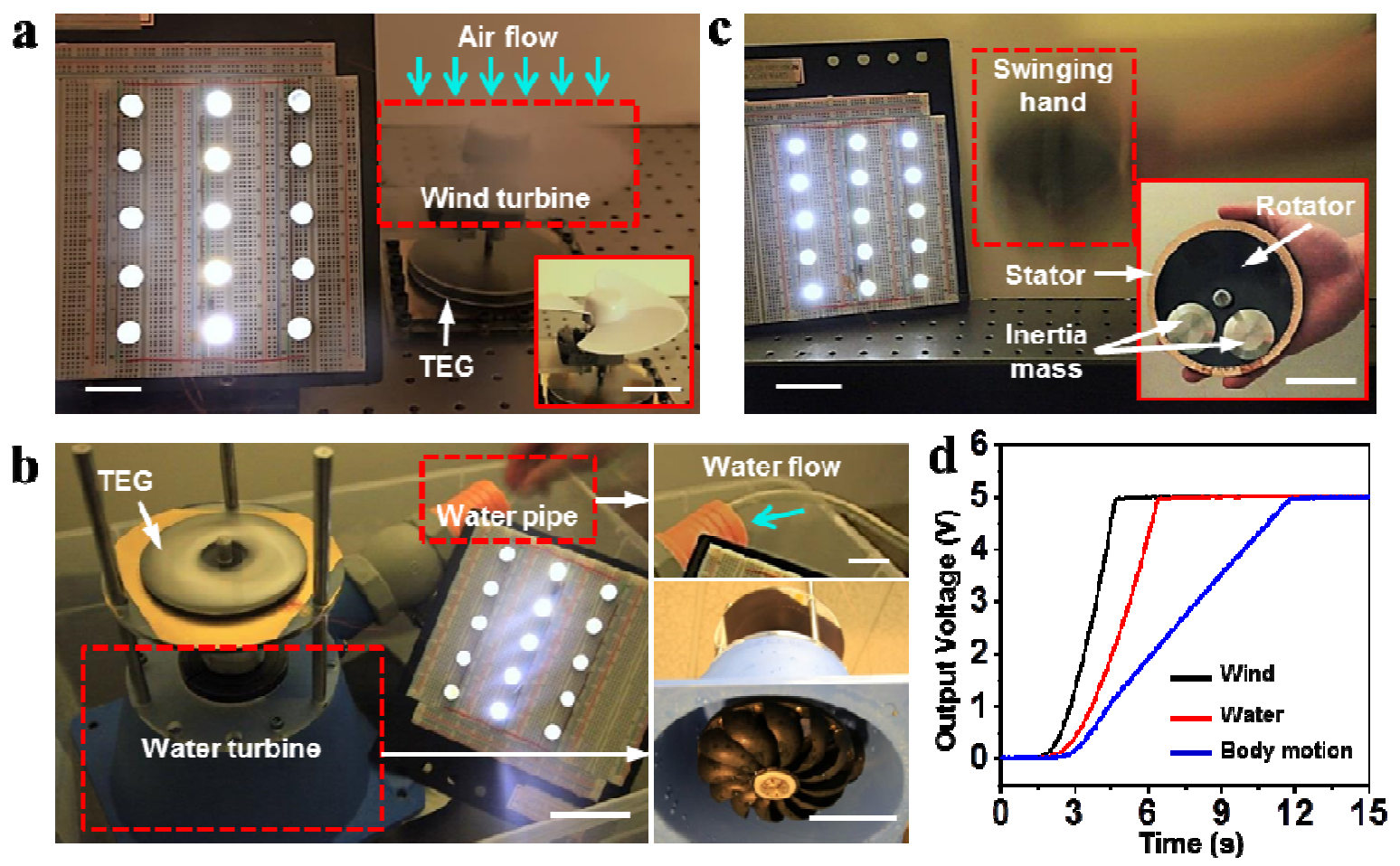

Figure 11. Demonstrations of a rotary TENG in harvesting ambient energy. (a) Harvesting energy from light wind at a flow speed of $6 \mathrm{~m} / \mathrm{s}$ by the TENG for powering an array of spot lights (scale bar: $3 \mathrm{~cm}$ ). Inset: a wind turbine that transmits torque to the TENG (scale bar: $5 \mathrm{~cm}$ ). (b) Harvesting energy from water flow at a flow rate of $5.5 \mathrm{~L} / \mathrm{min}$ by the TENG for powering an array of spot lights (scale bar: $5 \mathrm{~cm}$ ). Insets: tap water is directed into a water turbine through a water pipe (top; scale bar: $2 \mathrm{~cm}$ ); upward view of the water turbine (bottom; scale bar: $7 \mathrm{~cm}$ ). (c) Harvesting energy from body motion by the TENG that is being gently swung with a hand for powering an array of spot lights (scale bar: $5 \mathrm{~cm}$ ). Inset: the hand-held TENG with two pieces of inertia mass attached to the rotator (scale bar: $5 \mathrm{~cm}$ ). (d) Output voltage of the power-supplying system when the TENG is driven by the above three types of ambient mechanical energy. 

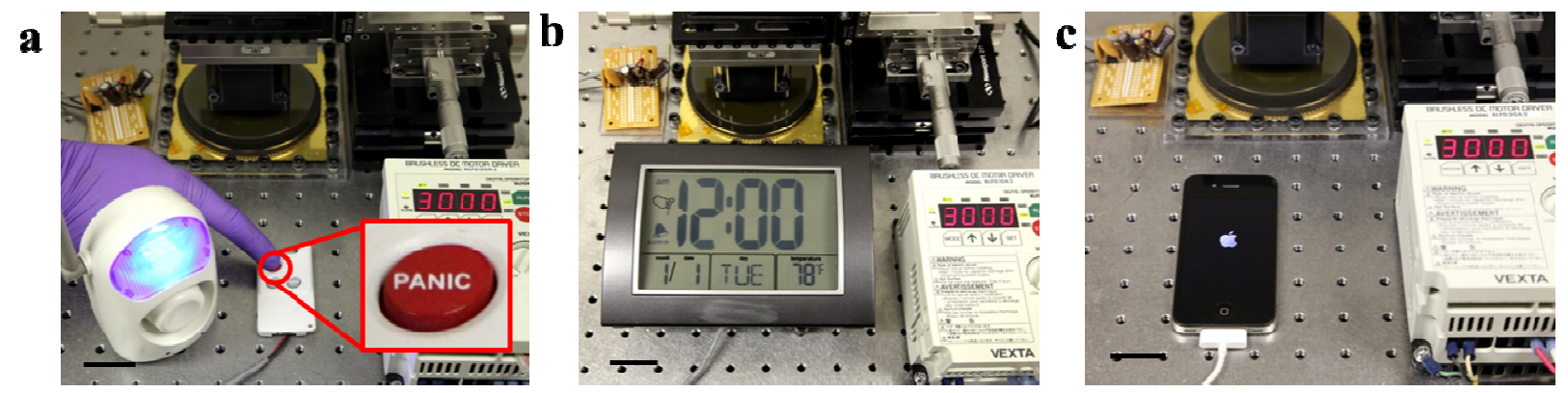

Figure 12. Demonstrations of the integrated power-supplying system for driving and charging electronics. (a) Photograph of an alarm triggered by a wireless emitter that relies on the powersupplying system (scale bar: $3 \mathrm{~cm}$ ). Inset: Photograph of the "panic" button that sets off the alarm. (b) Photograph of a multi-function digital clock driven by the power-supplying system (scale bar: $3 \mathrm{~cm}$ ). (c) Photograph of a cellphone that is being charged by the power-supplying system (scale bar: $3 \mathrm{~cm}$ ). 


\title{
Triboelectric Nanogenerators as a New Energy Technology: from Fundamentals, Devices, to Applications
}

\author{
Guang Zhu ${ }^{1, *}$, Peng, Bai ${ }^{2}$, Jun Chen ${ }^{2}$, Qingshen Jing ${ }^{2}$, Zhong Lin Wang ${ }^{1,2, *}$ \\ ${ }^{1}$ Beijing Institute of Nanoenergy and Nanosystems, Chinese Academy of Sciences, Beijing, \\ 100083, China. \\ ${ }^{2}$ School of Materials Science and Engineering, Georgia Institute of Technology, Atlanta, GA \\ 30332, USA.
}

*Correspondence to: zhuguang@binn.cas.cn, zlwang@binn.cas.cn

Keywords: contact electrification, self-powered electronics, energy harvesting, triboelectric nanogenerator

\begin{abstract}
Contact electrification is coupled with electrostatic induction in developing triboelectric nanogenerator as a new energy technology. The triboelectric nanogenerator has two basic operating modes that can be used to harvest a variety of mechanical energy. It provides not only a viable means of powering portable and wearable electronics, but also demonstrates a possible route towards power generation in large scale. This paper makes a comprehensive review on fundamentals, operating modes, device design and performance enhancement of this newly emerged technology.
\end{abstract}

